\title{
SOCIAL LEARNING NETWORKING DIGITAL AFFORDANCE DESIGN
}

\author{
Ben Chang and Rotua Zendrato \\ Institute of Learning and Instruction, National Central University, Taiwan \\ No. 300, Zhongda Rd., Zhongli District, Taoyuan City 32001, Taiwan (R.O.C.)
}

\begin{abstract}
Social networking platforms, such as Facebook, WeChat, and Line, are affecting and reshaping on how we think and what we communicate. This phenomenon reveals that the use of social networking platforms might not only be in private daily life but also in learning as well as teaching. For learning purpose on social networking platforms, we will argue that a learning activity needs a deliberate program and clear design principles. Based on the argument, in this article, we elaborate an idea named digital affordance and propose a digital affordance model following three types' practical activities.
\end{abstract}

\section{KEYWORDS}

Social Networking Platform, Learning Activity Design, Digital Affordance

\section{INTRODUCTION}

Tremendous students use social networking platforms for communicating and sharing thoughts every day. The phenomenon is inspiring researchers to consider how to apply social networking platforms to support learners' teaching and learning. Although traditional classrooms have many advantages including face-to-face lecturing, in-class small group discussions and so on, social networking platforms taking the advantages of multimedia, dynamic, instant, and ubiquitous computing can further provide traditional classrooms with pre-class, in-class and after-class learning activities for learners to exchange thoughts and ideas seamlessly. However, on social networking platforms for the learning purpose, teachers and students need well-designed learning activities since learning has its pedagogical and unique approaches. Obviously, it is worth to explore how to design a good instructional and learning approach on social networking platforms (Hung \& Yuen, 2010) so that the social networking platforms can be applied for learning purpose.

Affordance proposed by Gibson (1979) has been using over the decades in many disciplines (Blewett \& Hugo, 2016; You \& Chen, 2007; Hartson, 2003), which well explains how a learner is affected in an environment whatever it is in a natural environment or a manipulated environment. Social networking platforms reveal various new opportunities to traditional affordance settings, which inspire researchers considering the possibility of extending traditional affordance to digital affordance. To sum above, having learning activities on social networking platforms need well design and the aim of this paper is to explore a digital affordance model and its' possibility of digital affordance design in a social networking environment.

\section{DIGITAL AFFORDANCE}

As mentioned above, Gibson's affordance theory (1979) is well accepted, and now the technology evolution makes the traditional affordance settings can be digitalized. Compared with the traditional affordance, digital affordance has many advantages, such as dynamic, multimedia, anytime, anywhere and so on. Below, the attributes of digital affordance are elaborated from several perspectives. 


\subsection{Traditional Affordance vs. Digital Affordance}

Digital affordance compared to traditional affordance idea has many advantages. Figure 1 elaborates the advantages from the environment, affordance type, participant role, and participant location perspectives.

The differences between traditional and digital affordances are discussed below:

- Environment: The affordance settings in a traditional environment are used to be physical, fixed, and hard to change. However, the settings of a digital affordance on an online social networking platform can be very dynamic and easy to be re-arranged.

- Affordance Type: The traditional affordance due to physical constraint is usually practiced face-to-face, but the digital affordance can be either face-to-face or non-face-to-face.

- Participant Role: The participants in the traditional affordance environment should be real persons. However, in the digital affordance environment, the participants can be real users, remote users, or even non-real users, such as avatars and non-player characters created and manipulated by the computer.

- Participant Location: The participants' location in the traditional environment should be in the same place, but on a social networking platform, the affordance activities can occur in many different places simultaneously.

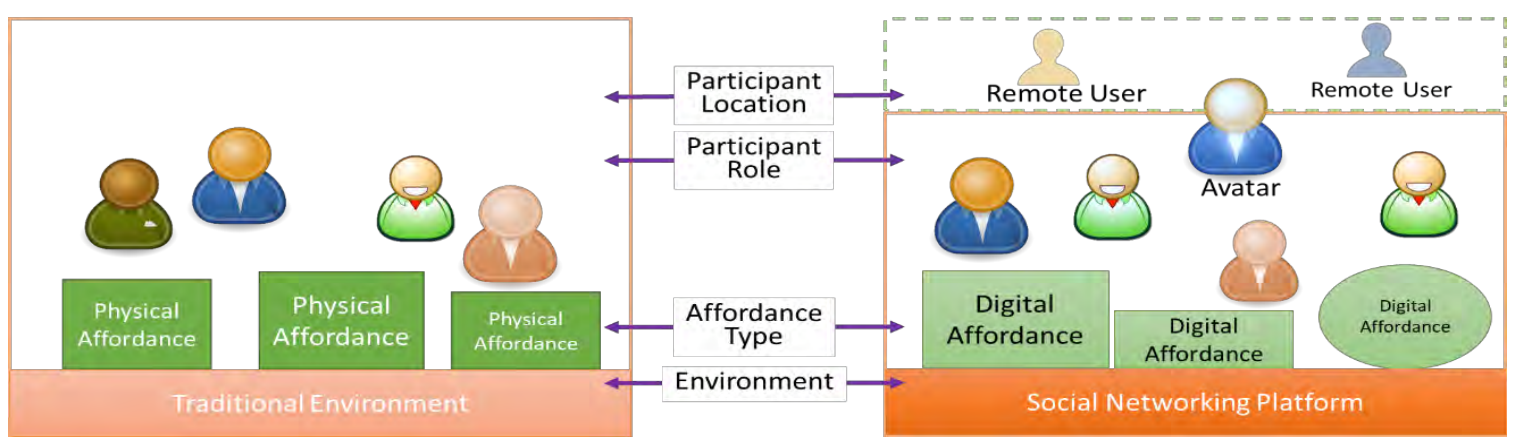

Figure 1. Affordance design in a traditional environment and social networking environment

\subsection{Digital Affordance Role in Social Learning Networking}

To illustrate how a digital affordance is applied in a social learning networking environment, a model with four layers including social networking platforms, basic tools, digital affordances, and learning activities is proposed. As shown in Figure 2, the first layer of the model is the social networking platforms layer. Various social networking platforms, such as Facebook, Line, and Twitter, are available for users to have interactive activities in this layer. Beyond the social networking platforms, as the second layer of the model, various basic tools with functions such as file uploading, photo taking, video exchanging, chatting, etc. support the activities occurred on the social networking platforms. Based on the basic tools layer, many designed digital affordances are available, and beyond the digital affordances, many learning activities are provided.

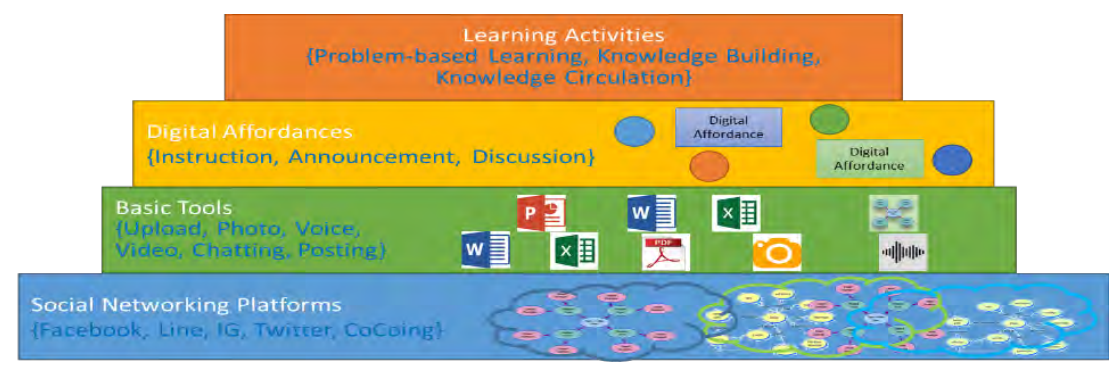

Figure 2. Social learning networking combining with digital affordance model 
As a digital affordance, there are several attributes illustrated below:

- The first attribute of a digital affordance is its GOAL SETTING or AGENDA SETTING. Goal setting or agenda setting attribute lets the teachers and the students can have a shared vision.

- The second attribute of digital affordance is TIME SEQUENCE. The time sequence can be pre-class, in-class or after-class.

- The third attribute of digital affordance is ASYNCHRONOUS or SYNCHRONOUS, which means that digital affordance can be designed as an asynchronous or synchronous activity.

- The fourth attribute of digital affordance is an INSTANT MESSAGE or NON-INSTANT MESSAGE. This means that the interaction among the users can be instant messages activity or non-instant messages activity.

Currently, only four digital affordance attributes are proposed. However, more attributes might be found in further exploration.

\subsection{Digital Affordance Examples}

To illustrate what is a digital affordance and how it operates, three examples are proposed below. These digital affordances design can be applied for social learning networking platforms to conduct learning activities.

\subsubsection{Type I: Idea Generation, Discussion, and Integration Activity}

As shown in Figure 3, type I example illustrates how a digital affordance is applied to support idea generation, discussion, and integration learning activity.

Pre-class activity

In this case, at the pre-class stage, the teachers or students need to set the learning goal or learning agenda. All the students at this stage generate as many ideas as possible non-face-to-face asynchronously on the social networking platform.

In-class activity

In the class, the students are assigned to their groups according to their pre-class discussion to discuss and to settles down their ideas. Each of the group students illustrates their idea at the end of the class. Instant message software can be applied in the in-class activity to support the students to incorporate their discussions of a topic.

After-class activity

After the class, all the students need to have some reflections on their social learning networking to extend the learning effect. The asynchronous discussion board can be applied at this stage.

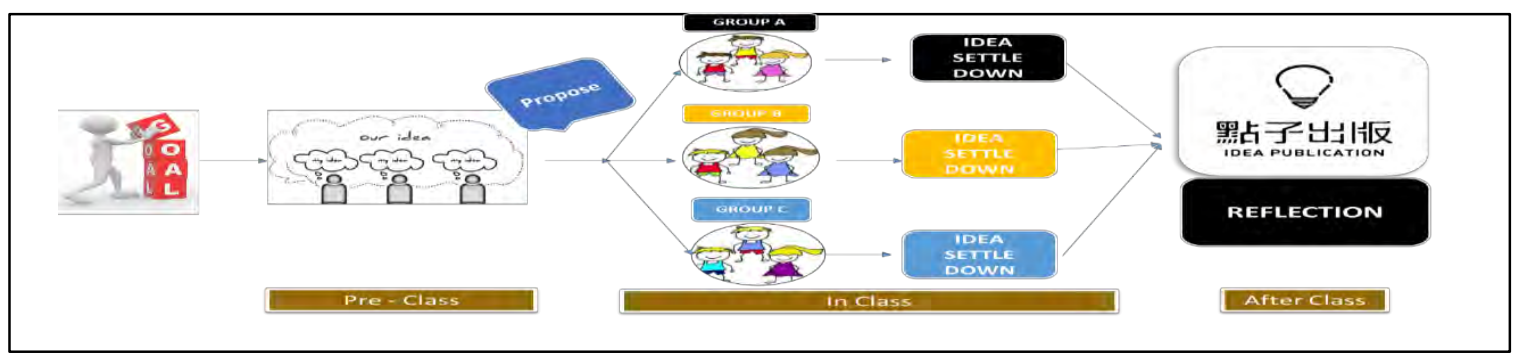

Figure 3. Type I: Idea generation, discussion, and integration activity

\subsubsection{Type II: Topic-oriented Discussion, Idea Settle Down, Meta-Analysis, and Reflection}

As shown in Figure 4, type II example illustrates how a digital affordance is applied in a topic-oriented discussion, settling down idea, meta-analysis, and reflection.

Pre-class activity

In the pre-class stage, the students are grouped, and they have their own topic to study in their group. Before the class, the students with their team members need to settle down their idea. Synchronous software like video conferencing or asynchronous software like discussion board can be applied in this stage. 
In-class activity

In the class, all the students elaborate their subject discussed in the group to others and try to integrate their subject into a complete one. Once they finish their topic discussion, they all have the meta-analysis activity on the complete topic together.

After-class activity

They all have the topic reflection in the after-class activity. Asynchronous software can support reflection.

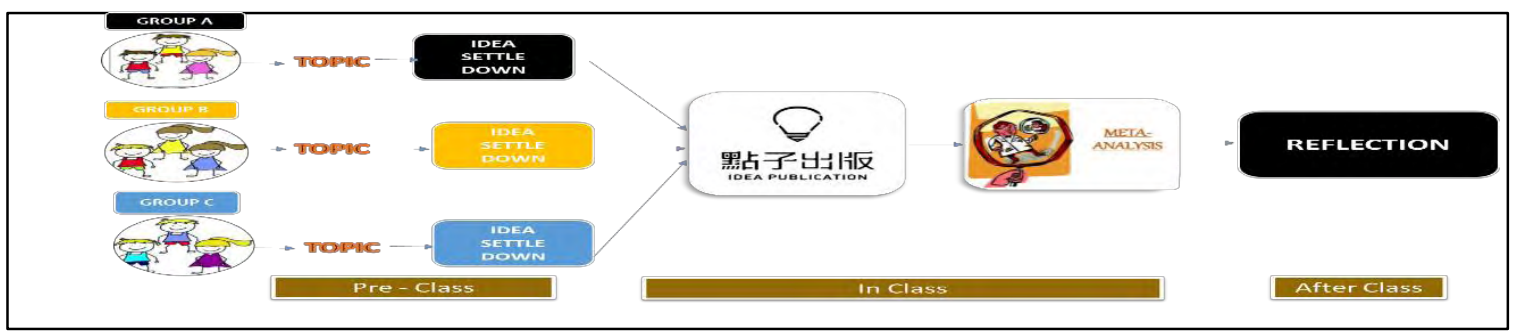

Figure 4. Type II: Topic-oriented discussion, idea settle down, meta-analysis, and reflection

\subsubsection{Type III: Topic Study following with Exercise, and Peer Review}

As shown in Figure 5, type III example illustrates how a digital affordance can support topic study, exercise, and peer review activity.

Pre-class activity

At the pre-class stage, the students acquire their domain knowledge by themselves. Through the self-learning activity, the students consolidate their background knowledge.

In-class activity

In the class, all the students study the topic and do the exercise together. The synchronous tool can be applied to have the coordinate activity.

After-class activity

After the class, the students reflect their topic discussion and exercise results.

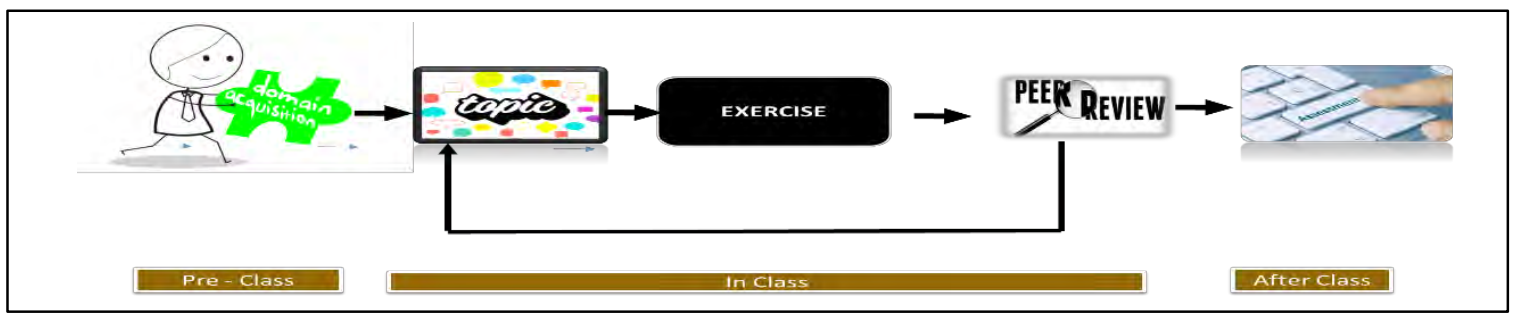

Figure 5. Type III: Topic study following with exercise, and peer review

\section{CONCLUSION}

Social networking platforms have become ubiquitous in our daily life, and have demonstrated their potential to assist with learning. However, applying social networking platforms for education and learning purposes need deliberate designs. In this study, the authors extend the traditional affordance concept to digital affordance. On the digital affordance concept, four attributes including goal/agenda setting, time sequence, asynchronous/synchronous, and instant/non-instant messages are discussed. Meanwhile, three digital affordance types are illustrated. Currently, the study focuses on digital affordance idea elaboration. In the future, these digital affordances need to be explored on social learning networking platforms and study their effects. 


\section{REFERENCES}

Blewett, C., \& Hugo, W. (2016). Actant affordances: A brief history of affordance theory and a Latourian extension for education technology research. Critical Studies in Teaching and Learning, 4(1), 55-76.

Gibson, J. J. (1979). The ecological approach to visual perception. New York: Houghton Mifflin.

Hartson, R. (2003). Cognitive, physical, sensory, and functional affordances in interaction design. Behaviour \& Information Technology, 22(5), 315-338.

You, H. C., \& Chen, K. (2007). Applications of affordance and semantics in product design. Design Studies, 28(1), 23-38. 